\title{
Commentary on The Case Formulation Approach to Psychotherapy Research Revisited
}

\section{Who Needs a Case Formulation and Why: Clinicians Use the Case Formulation to Guide Decision-Making}

\author{
JACQUELINE B. PERSONS ${ }^{\mathrm{a}, \mathrm{b}}$ \\ a San Francisco Bay Area Center for Cognitive Therapy and University of California at Berkeley \\ Correspondence regarding this article should be addressed to Jacqueline B. Persons, San Francisco Bay Area \\ Center for Cognitive Therapy, 5435 College Avenue, Oakland, CA 94618. \\ Email: jbp@sfbacct.com
}

\begin{abstract}
Protocols for empirically-supported treatments (ESTs) typically do not require the therapist to develop an individualized formulation of the patient, nor do they describe procedures for doing this. I offer some hypotheses about why the idiographic case formulation receives short shrift in most EST protocols, I describe an exception to this general rule, and I conclude with a discussion of implications of these ideas for psychotherapy research.
\end{abstract}

Key words: idiographic case formulation; treatment developers; treatment utility; clinical decisionmaking; randomized trial; empirically supported treatments; clinical case studies; case studies

As Tracy Eells (Eells, 2013) points out in his very interesting examination of the role of case formulation in psychotherapy research, clinicians view the case formulation as essential to their work, but psychotherapy treatment developers do not typically place much value on the formulation. Protocols for empirically-supported treatments (ESTs) typically do not include procedures to guide the therapist to develop an idiographic (individualized) formulation of each case. This was true 20 years ago (Persons, 1991), and, as (Eells, 2013) points out, remains true now. The discrepancy between clinicians' and researchers' views of the importance of the case formulation is an example of the oft-discussed scientist-practitioner gap and of the under-focus on assessment described by (Hunsley \& Mash, 2007).

I offer some hypotheses about why most EST protocols do not call for the therapist to develop an idiographic case formulation, I describe an exception to this general rule, and I discuss implications of these ideas for psychotherapy research.

\section{WHY THE IDIOGRAPHIC CASE FORMULATION GETS SHORT SHRIFT IN EST PROTOCOLS}

Protocols for empirically-supported treatments (ESTs) typically do not call for the therapist to develop an idiographic (individualized) formulation of each case. At the same time, it is important to recognize that psychotherapy protocols do include a formulation in two ways. 
Who Needs a Case Formulation and Why: Clinicians Use

the Case Formulation to Guide Decision-Making

J.B.Persons

Pragmatic Case Studies in Psychotherapy, http://pcsp.libraries.rutgers.edu

Volume 9, Module 4, Article 4, pp. 448-456, 12-12-13 [copyright by author]

First, all treatments are based on a nomothetic, or general, formulation. The ESTs are generally disorder-focused treatments, and they are based on a formulation of the disorder that underpins the interventions described in the protocol. For example, panic control treatment (Barlow \& Craske, 2000) is based on the conceptualization that symptoms of panic disorder result from anxiety sensitivity, or catastrophic misinterpretations of benign somatic sensations (Goldstein \& Chambless, 1978), and the interventions in the protocol (interoceptive exposure, cognitive restructuring, psychoeducation), target that mechanism. Thus in an important way, the EST protocols are guided by a formulation, albeit a nomothetic formulation of the disorder the EST treats, not an idiographic formulation of the individual case.

In addition, many EST protocols include procedures to identify some of the idiographic details of the nomothetic formulation. For example, the panic control treatment protocol includes assessment procedures to help patient and therapist identify which somatic sensations are particularly frightening for each individual patient so they can be targeted in treatment. Similarly, cognitive therapy for depression (Beck, Rush, Shaw, \& Emery, 1979) assigns a behavioral activity schedule as a first homework assignment; the activity schedule assignment is designed to help the therapist identify some of the idiographic behavioral aspects of the patient's depressive symptoms.

Nevertheless, the typical EST protocol does not require the therapist to develop a complete idiographic formulation of the disorder the protocol treats. For example, Beck's cognitive therapy protocol (Beck et al., 1979) does not include systematic procedures to guide the therapist to identify which particular automatic thoughts, behaviors, and schemas underpin a patient's depressive symptoms.

I speculate that EST protocols do not include procedures to help the therapist develop a complete idiographic formulation for several reasons, all related to the fact that the treatment developer and the clinician have different goals and needs. One difference between treatment developers and clinicians is that treatment developers typically focus on disorders, whereas clinicians focus on patients. The treatment developer works to devise a therapy that will bring the average case of the disorder targeted by the treatment to remission. The clinician has a different goal, and that is to help the patient who is in his/her office at that moment reduce suffering, improve functioning, and accomplish his or her idiographic goals, and to do this via treatment that is consistent with his or her preferences and values (Sackett, Richardson, Rosenberg, \& Haynes, 1997). Often the person has multiple disorders as well as other difficulties (e.g., marital problems, medical substance abuse, unemployment) that are not disorders but that directly or indirectly cause suffering, impede functioning, and that may interfere with treatment. As a result, the clinician is focused on multiple disorders and problems, whereas usually the treatment developer is usually focused on a single disorder.

Moreover, the treatment developer is focused on the goal of bringing the disorder to remission, and the clinician is focused on the goal of helping the patient have a better quality of life and meet idiographic goals. A not uncommon example is the patient who wants to overcome shyness so he can date and find a partner. Although this patient may meet criteria for social anxiety disorder, he does not have the goal of treating his social anxiety disorder to remission. 
Who Needs a Case Formulation and Why: Clinicians Use

the Case Formulation to Guide Decision-Making

J.B.Persons

Pragmatic Case Studies in Psychotherapy, http://pcsp.libraries.rutgers.edu

Volume 9, Module 4, Article 4, pp. 448-456, 12-12-13 [copyright by author]

The divergent goals of the clinician and the treatment developer mean that the clinician is confronted with many more decisions than the treatment developer or even the clinician who is implementing the disorder-focused treatment. The clinician who is treating panic disorder must make several decisions, including which somatic sensations to target in treatment. The clinician treating "Jane," who has three anxiety disorders and a mood disorder and a marital problem and abuses alcohol, must make a large number of difficult decisions about what treatment targets to address in what order to help Jane accomplish her goals. An idiographic case formulation helps the therapist make these decisions. The formulation is a hypothesis that describes, among other things, the relationships among Jane's various problems. For example, the formulation might hypothesize that Jane desperately wants to keep her marriage and that her husband derives important meaning in his life by driving Jane wherever she needs to go. This formulation has important implications for treatment, and suggests that a full-bore attack on Jane's panic symptoms without regard for the implications on her marriage of her recovery from those symptoms is not likely to be acceptable to Jane or very successful.

Typical decisions the clinician confronts that the EST treatment developer does not confront include: When there are multiple disorders, ought I target one disorder at a time, or might several or even all of the disorders be maintained by common psychological mechanisms, so that if I identify and focus treatment on those mechanisms, I can treat more than one disorder simultaneously? What is the best way to proceed if the patient has goals other than treating a DSM disorder to remission? If several ESTs are available to treat the patient's primary disorder, how do I select one? Must I rely on one EST protocol or might I draw interventions from more than one? What is the best way to proceed if the patient does not comply with treatment? What is the best way to proceed if the patient does not respond to treatment?

The clinician places a high value on the idiographic case formulation because it helps him/her answer all of these questions (Persons, 2005, 2008). The model of case formulationdriven or psychotherapy (Persons, 2006) and Figure 2 of Eells (2013) describes a method of clinical work that guides the therapist's decision-making in all the situations just described. The model calls for the clinician to collect assessment data to develop an idiographic formulation of the case that describes relationships among the patient's various problems and disorders, and identifies psychological mechanisms (e.g., distorted schemas, perfectionism, anxiety sensitivity) that cause and maintain those problems. Then the therapist uses the case formulation to select interventions from a wide variety of sources, including multiple ESTs, to target the mechanisms. While implementing the treatment, the therapist collects data to evaluate the process (e.g., is the patient accepting and using the interventions I am offering?) and outcome of treatment at every session. The progress monitoring data assess outcome by evaluating the effects of the treatment on both the patient's symptoms and functioning, and also on the mechanisms the treatment is targeting, thus providing a test of the formulation hypothesis. If the data indicate that outcome is poor, many options are available to the therapist, but one is to revisit the formulation to correct any inaccuracies and consider whether a revised formulation might suggest new interventions that might be more helpful to the patient.

To summarize, because the treatment developer and clinician have different goals and needs, and because the clinician, to accomplish his/her goals must make many more decisions 
Who Needs a Case Formulation and Why: Clinicians Use

the Case Formulation to Guide Decision-Making

J.B.Persons

Pragmatic Case Studies in Psychotherapy, http://pcsp.libraries.rutgers.edu

Volume 9, Module 4, Article 4, pp. 448-456, 12-12-13 [copyright by author]

than the treatment developer and needs guidance to do this, the case formulation is much more valuable to the clinician than to the treatment developer. A similar observation was made by (Haynes, Leisen, \& Blaine, 1997), who argued that the idiographic case formulation was particularly useful in the treatment of complex cases.

Clinicians and researchers also have different needs in that most clinicians must be generalists to some degree, whereas the treatment developer can be a specialist. The clinician needs a tool box of procedures that $\mathrm{s} /$ he can use to meet the needs of the large variety of patients s/he sees over the course of the week or even the day. Instead, the treatment developer thinks intensively about a particular disorder or set of problems. Thus, Hinton and colleagues (Hinton, Pham, Tran, Safren, \& Otto, 2004; Hinton, Rivera, Hofmann, Barlow, \& Otto, 2012) developed a new treatment, Culturally Adapted-CBT for PTSD, for traumatized ethnic and refugee groups. The treatment addresses the particular challenges of treating this group of individuals in several ways, including by presenting the material in a way that is easily understood by those with limited English and education, emphasizing the treatment of somatic sensations, and targeting the anger and comorbid anxiety disorders that these individuals commonly experience. This treatment is an outstanding contribution to this group of patients and to the clinicians who see them frequently. But it is not very helpful to the average clinician, who simply cannot learn a new protocol for a specialized population that s/he rarely encounters. The treatment developer's response to unique symptom presentations is to develop a new treatment; this strategy leads to a proliferation of new treatments that is overwhelming to the clinician, and is particularly frustrating when the new treatments consist of adaptations of currently-available treatments. As (Hong, 2013; Zayfert, 2008) point out, an alternative to developing a new treatment for patients who have unique cultural or ethnic backgrounds is to use a case formulation-driven approach to treatment, incorporating information about cultural and related factors into the formulation and using the formulation to guide treatment adaptation to address those factors. In fact, the case formulation approach can help the therapist attend to any unique idiographic factor that the patient brings to the treatment situation, including such things as physical handicap, medical illness, poverty, sexual orientation, or other factors. Using the idiographic case formulation as a way to identify and guide treatment to address the unique features of a patient's presentation provides the clinician, who must be a generalist, much more assistance than does a shelf-full of EST protocols that address specialized patient populations.

\section{AN EXCEPTION TO THE GENERAL RULE}

A very interesting exception to the general rule that treatment developers do not build procedures for obtaining and using an individualized case formulation into the protocol is the modular treatment for children and adolescents with mood, conduct and anxiety disorders recently developed by Bruce Chorpita and John Weisz and colleagues (Chorpita \& Weisz, 2005). The modular treatment, dubbed Modular Approach to Therapy for Children with Anxiety, Depression, or Conduct Problems (MATCH), consists of a group of intervention modules, (e.g., for self-calming, modifying negative cognitions, and increasing compliance with parents' directives) that therapists, guided by decision flowcharts, select to meet the idiographic needs of the child they are treating at that moment. The decision flowcharts serve as a sort of rudimentary case formulation; they serve one of the key functions of the case formulation, namely to guide 
Who Needs a Case Formulation and Why: Clinicians Use

the Case Formulation to Guide Decision-Making

J.B.Persons

Pragmatic Case Studies in Psychotherapy, http://pcsp.libraries.rutgers.edu

Volume 9, Module 4, Article 4, pp. 448-456, 12-12-13 [copyright by author]

decision-making and, in particular, intervention selection, to help the therapist individualize the treatment for the complex case of a patient with multiple comorbidities. These flowcharts are needed because the therapist must make decisions about implementing interventions for multiple-problem patients, and needs some sort of guidance (a decision flowchart, or case formulation) as s/he does this. A recent randomized trial (Weisz et al., 2012) showed that patients who received modular treatment fared better on several dependent variables than those who received standard treatment (ESTs for anxiety, mood, or conduct problems) or usual care.

\section{IMPLICATIONS FOR PSYCHOTHERAPY RESEARCH}

The notion that the major function of the case formulation is to help clinicians make decisions for complex cases has several implications for psychotherapy research.

First, because the treatment utility of the idiographic case formulation is greatest when the case is complex and the clinician confronts many decisions, those situations are the perfect petri dish for studying and demonstrating the treatment utility of the idiographic case formulation. The study by (Weisz et al., 2012) reviewed above exemplifies this point, as does a study by (Johansson, 2012) of individualized versus standardized treatment of depression and comorbid disorders. Other examples include the single case studies of treatment failure by (Persons, Beckner, \& Tompkins, 2013; Persons \& Mikami, 2002), who showed that when progress monitoring data indicate that the patient is not responding to treatment, the strategy of reviewing and revising the case formulation to develop a new one can lead to new interventions that can lead to a good outcome.

Second, single case designs have great potential to contribute to science generally and this area of study in particular (Fishman, 2002, 2005; Hayes, Barlow, \& Nelson-Gray, 1999). Some of the most compelling data in support of the treatment utility of the case formulation have come from single cases (e. g., (Iwata et al., 1994 )).

Third and related, clinicians have an opportunity, even a responsibility, to make contributions to the research demonstrating the treatment utility of the idiographic case formulation. Their daily experience informs them about the need and utility of the case formulation, and the scientist-practitioners among them can make needed contributions to the research literature based on these observations. Single case studies offer a good way for clinicians to do this, and publication outlets for this work are available-including of course, this journal.

Fourth, a treatment consisting of modules and a decision tree or formulation module that helps the therapist select intervention modules and make other clinical decisions, has great promise. Modules, or what (Embry \& Biglan, 2008) and called "kernels," that is, "fundamental units of behavioral influence that appear to underlie effective prevention and treatment for children, adults, and families” (Embry \& Biglan, 2008) (p. 75) consists of single procedures or interventions, such as verbal praise and the Premack principle. "Kernels" or modules differ from “ears” (complete protocols; (Weisz, Ugueto, Herren, Afienko, \& Rutt, 2011)). The development of modular treatments can narrow the scientist-practitioner gap by bringing the psychotherapy 
Who Needs a Case Formulation and Why: Clinicians Use

the Case Formulation to Guide Decision-Making

J.B.Persons

Pragmatic Case Studies in Psychotherapy, http://pcsp.libraries.rutgers.edu

Volume 9, Module 4, Article 4, pp. 448-456, 12-12-13 [copyright by author]

treatment literature closer to the way clinicians now work, by providing an evidence base for the "kernels" that clinicians are routinely pulling out of the "ears" of the EST protocols, and by providing therapists with flexible treatments that can be adapted to treat a wide variety of patients.

The "kernels" idea can be used to dismantle the elements of the case formulation-driven mode of work described above and depicted in Figure 2 in (Eells, 2013). Thus, psychotherapy treatment developers can develop and study kernels for conducting an initial assessment, developing an initial mechanism hypothesis, informing the patient about the case formulation, using the formulation to guide intervention, monitoring progress, using the progress monitoring data to overcome lack of progress, and so on.

Several investigators have already developed progress monitoring kernels (e.g., (Miller, Duncan, \& Brown, 2006; Persons, Koerner, Thomas, \& Liu, 2012)). Probably the one with the strongest empirical support is the OQ-45 developed by Michael Lambert and his colleagues, who have shown in randomized trials that use of the OQ-45 leads to improved outcomes, especially for patients who have an initial poor response to treatment (Lambert, Harmon, Slade, Whipple, \& Hawkins, 2005). My colleagues and I at the San Francisco Bay Area Center for Cognitive Therapy have developed a feedback kernel, the Session Assignment and Feedback Form (SAFF), that the therapist can use to track the patient's homework assignments and to collect feedback from the patient about the session and the therapy (Persons, Hong, Lemle Beckner, Owen, \& Eidelman, 2012). We are collecting some data to test the hypothesis that its use, paired with symptom monitoring data, is associated with improved outcomes.

Consistent with the modular approach to treatment development, investigators have begun to develop interventions that target transdiagnostic mechanisms, such as intolerance of uncertainty (Buhr \& Dugas, 2006) and perfectionism (Glover, Brown, Fairburn, \& Shafran, 2007), rather than EST disorders. (Frank \& Davidson, in press) offer an excellent overview of this literature for the clinician.

Finally, the observation that clinicians are consistently convinced of the importance of an idiographic case formulation to guide their decision-making suggests that studies of clinicians' adoption of evidence-based practices (EBP) are likely to show that adoption is greater when the EBP consists of kernels or modules, including an idiographic case formulation module that the therapist uses to guide selection of modules and make other clinical decisions in order to flexibly carry out treatment to meet the needs of the unique individual who is in the therapist's office at that moment.

Finally, the observation that clinicians are consistently convinced of the importance of an idiographic case formulation to guide their decision-making is important to highlight. For this observation suggests that studies of clinicians' adoption of evidence-based practices (EBP) are likely to show that adoption is greater when the EBP consists of kernels or modules, including an idiographic case formulation module that the therapist uses to guide selection of modules and make other clinical decisions in order to flexibly carry out treatment to meet the needs of the unique individual who is in the therapist's office at that moment. 


\section{REFERENCES}

Barlow, D. H., \& Craske, M. G. (2000). Mastery of your anxiety and panic: Client workbook for anxiety and panic. San Antonio, TX: Graywind Psychological Corporation.

Beck, A. T., Rush, J. A., Shaw, B. F., \& Emery, G. (1979). Cognitive therapy for depression. New York: Guilford Press.

Buhr, K., \& Dugas, M. J. (2006). Investigating the construct validity of intolerance of uncertainty and its unique relationship with worry. Journal of Anxiety Disorders, 20(2), 222-236.

Chorpita, B. F., \& Weisz, J. R. (2005). Modular approach to therapy for children with anxiety, depression, or conduct problems. Honolulu, HI/Boston, MA: University of Hawaii at Manoa/Judge Baker Children's Center, Harvard Medical School.

Eells, T. D. (2013). The case formulation approach to psychotherapy revisited. Pragmatic Case Studies in Psychotherapy, 9 (4), Article 3, 426-447. Available: http://hdl.rutgers.edu/1782.1/pcsp_journal

Embry, D. D., \& Biglan, A. (2008). Evidence-based kernels: Fundamental units of behavioral influence. Clinical Child Family Psychology Review, 11, 75-113.

Fishman, D. B. (2002). From single case to database: A new method for enhancing psychotherapy, forensic, and other psychological practice. Applied \& Preventive Psychology, 10, 275-304.

Fishman, D.B. (2005). Editor's introduction to PCSP--From single case to database: A new method for enhancing psychotherapy practice. Pragmatic Case Studies in Psychotherapy, 1(1), Article 2, 1-50. Available: http://hdl.rutgers.edu/1782.1/pcsp_journal

Frank, R. I., \& Davidson, J. (in press). A transdiagnostic roadmap to case formulation and treatment planning: Practical guidance for clinical decision making. Oakland, CA: New Harbinger Publications, Inc.

Glover, D. S., Brown, G. P., Fairburn, C. G., \& Shafran, R. (2007). A preliminary evaluation of cognitive-behaviour therapy ffor clinical perfectionism: A case series. British Journal of Clinical Psychology, 46, 85-94.

Goldstein, A., \& Chambless, D. L. (1978). A reanalysis of agoraphobia. Behavior Therapy, 9, 47-59.

Hayes, S. C., Barlow, D. H., \& Nelson-Gray, R. O. (1999). The scientist-practitioner: Research and accountability in the age of managed care. (2nd ed.). Boston, MA: Allyn and Bacon.

Haynes, S. N., Leisen, M. B., \& Blaine, D. D. (1997). Design of individualized behavioral treatment programs using functional analytic clinical case models. Psychological Assessment, 9, 334-348.

Hinton, D. E., Pham, T., Tran, M., Safren, S. A., \& Otto, M. W. (2004). CBT for Vietnamese refugees with treatment-resistant PTSD and panic attacks: A pilot study. Journal of Traumatic Stress, 17(5), 429-433.

Hinton, D. E., Rivera, E. I., Hofmann, S. G., Barlow, D. H., \& Otto, M. W. (2012). Adapting CBT for traumatized refugees and ethnic minority patients: Examples from culturally adapted CBT (CA-CBT). Transcultural Psychiatry, 49(2), 340-365.

Hong, J. J. (2013). An idiographic evidence-based approach to addressing cultural factors in treatment: A case example. the Behavior Therapist, 36(6), 143-146. 
Who Needs a Case Formulation and Why: Clinicians Use

the Case Formulation to Guide Decision-Making

J.B.Persons

Pragmatic Case Studies in Psychotherapy, http://pcsp. libraries.rutgers.edu

Volume 9, Module 4, Article 4, pp. 448-456, 12-12-13 [copyright by author]

Hunsley, J., \& Mash, E. J. (2007). Evidence-based assessment. Annual Review of Clinical Psychology, 3(29-51).

Iwata, B. A., Pace, G. M., Dorsey, M. F., Zarcone, J. R., Vollmer, T. R., \& Smith, R. G. (1994 ). The functions of self-injurious behavior: An experimental-epidemiological analysis. Journal of Applied Behavior Analysis(27), 215-240.

Johansson, R., Sjöberg, E., Sjögren, M., Johnsson, E., Carlbring, P., Andersson, T., Rousseau, A., Andersson, G.,. (2012). Tailored vs. standardized internet-based cognitive behavior therapy for depression and comorbid symptoms: A randomized controlled trial. Plos Clinical Trials, 7(5).

Lambert, M. J., Harmon, C., Slade, K., Whipple, J. L., \& Hawkins, E. J. (2005). Providing feedback to psychotherapists on their patients' progress: Clinical results and practice suggestions. Journal of Clinical Psychology, 61, 165-174.

Miller, S. D., Duncan, B. L., \& Brown, G. S. (2006). Using formal client feedback to improve retention and outcome. Journal of Brief Therapy, 5, 5-22.

Persons, J. B. (1991). Psychotherapy outcome studies do not accurately represent current models of psychotherapy: A proposed remedy. American Psychologist, 46, 99-106.

Persons, J. B. (2005). Empiricism, mechanism, and the practice of cognitive-behavior therapy. Behavior Therapy, 36, 107-118.

Persons, J. B. (2006). Case formulation-driven psychotherapy. Clinical Psychology: Science and Practice, 13, 167-170.

Persons, J. B. (2008). The case formulation approach to cognitive-behavior therapy. New York: Guilford.

Persons, J. B., Beckner, V. L., \& Tompkins, M. A. (2013). Testing case fomulation hypotheses in psychotherapy: Two case examples. Cognitive and Behavioral Practice, 20(4), 399409.

Persons, J. B., Hong, J., Lemle Beckner, V., Owen, D., \& Eidelman, P. (2012). Monitoring therapy process at every session: Development of a new tool and clinical examples of its utility. Presentation at the annual meeting of the Association for Behavioral and Cognitive Therapies, Naval Harbor, MD.

Persons, J. B., Koerner, K., Thomas, C., \& Liu, H. (2012). Can we train psychotherapists to monitor their clients' progress at every therapy session? Initial findings. Presentation at the annual meeting of the Association for Behavioral and Cognitive Therapies, National Harbor, MD.

Persons, J. B., \& Mikami, A. Y. (2002). Strategies for handling treatment failure successfully. Psychotherapy: Theory/Research/Practice/Training, 39, 139-151.

Sackett, D. L., Richardson, W. S., Rosenberg, W., \& Haynes, R. B. (1997). Evidence-based medicine: How to practice and teach EBM. New York: Churchill Livingstone.

Weisz, J. R., Chorpita, B. F., Palinkas, L. A., Schoenwald, S. K., Miranda, J., Bearman, S. K., et al. (2012). Testing standard and modular designs for psychotherapy treating depression, anxiety, and conduct problems in youth. Archives of General Psychiatry, 69(3), 274-282.

Weisz, J. R., Ugueto, A. M., Herren, J., Afienko, A. R., \& Rutt, C. (2011). Kernels vs. ears and other questions for a science of treatment dissemination Clinical Psychology: Science and Practice, 18(1), 41-46. 
J.B.Persons

Pragmatic Case Studies in Psychotherapy, http://pcsp.libraries.rutgers.edu

Volume 9, Module 4, Article 4, pp. 448-456, 12-12-13 [copyright by author]

Zayfert, C. (2008). Culturally competent treatment of posttraumatic stress disorder in clinical practice: An ideographic, transcultural approach. Clinical Psychology: Science and Practice, 15, 68-73. 